\title{
Longitudinal and transverse dynamics of ions from residual gas in an electron accelerator
}

\author{
A. Gamelin, ${ }^{*}$ C. Bruni, ${ }^{\dagger}$ and D. Radevych ${ }^{\ddagger}$ \\ LAL, Univ. Paris-Sud, CNRS/IN2P3, Université Paris-Saclay, Orsay 91898, France
}

(Received 13 February 2018; published 25 May 2018)

\begin{abstract}
The ion cloud produced from residual gas in an electron accelerator can degrade machine performances and produce instabilities. The ion dynamics in an accelerator is governed by the beam-ion interaction, magnetic fields and eventual mitigation strategies. Due to the fact that the beam has a nonuniform transverse size along its orbit, the ions move longitudinally and accumulate naturally at some points in the accelerator. In order to design effective mitigation strategies it is necessary to understand the ion dynamics not only in the transverse plane but also in the longitudinal direction. After introducing the physics behind the beam-ion interaction, we show how to get accumulation points for a realistic electron storage ring lattice. Simulations of the ion cloud dynamics, including the effect of magnetic fields on the ions, clearing electrodes and clearing gaps are shown. Longitudinal ion trapping due to the magnetic mirror effect in the dipole fringe fields is also detailed. Finally, the effectiveness of clearing electrode using longitudinal clearing fields is discussed and compared to clearing electrodes producing transverse field only.
\end{abstract}

DOI: 10.1103/PhysRevAccelBeams.21.054401

\section{INTRODUCTION}

In electron accelerators, residual gas can be ionized by several effects such as collisions with the electron beam or synchrotron radiation. The positive ions resulting from the ionization can be trapped in the negative beam potential of the electron beam [1]. The ion cloud effects on the beam can be divided in two groups. First, the effects of the electric field produced by the ion cloud distribution. The focusing force produced by the ions changes nonlinearly with the distance of the electron from the center of the ion cloud. This can lead to tune shift, tune spread, halo increase and emittance blow-up [2]. Second, the ion cloud oscillates at high frequency within the electron beam potential. As the electron beam feels an attractive force toward the ion cloud, the electron beam also oscillates which brings a resonant excitation of both oscillations. Under certain conditions the oscillations can grow exponentially and lead to an instability like the conventional ion trapping instability (CIT) [3] or the fast beam-ion instability (FBII) [4,5].

For these reasons it is crucial to decrease the number of ions. Techniques which allow to achieve this depends on accelerator type. The vacuum has to be as good as possible

\footnotetext{
*gamelin@1al.in2p3.fr

bruni@lal.in2p3.fr

radevych@uwm.edu
}

Published by the American Physical Society under the terms of the Creative Commons Attribution 4.0 International license. Further distribution of this work must maintain attribution to the author(s) and the published article's title, journal citation, and DOI. as beam degradation is directly proportional to the number of ions. But even a vacuum as good as a few $10^{-10} \mathrm{mbar}$ does not guarantee the absence of important effects on the beam. Clearing gaps provide an effective mitigation. It involves short periods of time during which the ions do not feel the electron beam focusing force. During this time the ions are free to drift away from the beam position. This technique has been used successfully in many accelerators like SPEAR3, NSLS-II or KEK-PF to avoid multiturn trapping or to fight against FBII $[6,7,3]$. The use of clearing electrodes in electron rings as a mitigation technique against ions is well documented here [8,9]. Clearing electrodes produce a constant electric field which extracts the ions from the beam potential well. In most cases a DC voltage of 2 or $3 \mathrm{kV}$ is enough to extract the ions from the beam potential well. Usually storage rings and pulsed linacs use clearing gaps [6] whereas Energy Recovery Linacs (ERLs) and small rings use clearing electrodes [10,11].

To design effective mitigation strategies against ions one has to understand the ion dynamics inside the accelerator. Foremost this dynamics depends strongly on the magnetic fields along the machine. Ions can be trapped by dipoles, under certain conditions, by the magnetic mirror effect [12]. A clearing gap duration and frequency should be chosen suitably for the ions to be able to escape the magnetic trapping. Also, the beam-ion interaction has been shown to be a 3D effect and its longitudinal component leads to ion accumulation points in the accelerator $[13,2]$. As a consequence, before positioning clearing electrodes a study of ion accumulation points should be done in order to maximize clearing efficiency. 
TABLE I. ThomX storage ring design parameters.

\begin{tabular}{lcc}
\hline \hline Parameter & Value & Units \\
\hline Beam energy & 50 & $\mathrm{MeV}$ \\
Emittance (geometrical) $\epsilon_{x, y}$ & 50 & $\mathrm{~nm} . \mathrm{rad}$ \\
Relative energy spread $\sigma_{\epsilon}$ & 0.6 & $\%$ \\
Bunch length at injection (rms) & 7 & $\mathrm{ps}$ \\
Bunch charge & 1 & $\mathrm{nC}$ \\
Circumference & 18 & $\mathrm{~m}$ \\
Revolution frequency & 16.7 & $\mathrm{MHz}$ \\
Number of bunch & 1 & \\
Current & 16.7 & $\mathrm{~mA}$ \\
Storage time before extraction/injection & 20 & $\mathrm{~ms}$ \\
Clearing gap duration & 4 & $\mu \mathrm{s}$ \\
Clearing electrode voltage & 500 & $\mathrm{~V}$ \\
Total pressure & $3 \times 10^{-10}$ & $\mathrm{mbar}$ \\
Partial pressure of CO & 10 & $\%$ of total \\
Partial pressure of $\mathrm{H}_{2}$ & 90 & $\%$ of total \\
\hline \hline
\end{tabular}

This paper deals with the ion dynamics in an electron accelerator combined to mitigation strategies. In the first part of this paper, the model used to describe the 3D beam-ion interaction is explained and its limitations are discussed. The ion accumulation conditions from the longitudinal component of the beam-ion interaction are discussed. The ion motion in dipole magnets is described and illustrated by simulations, the magnetic mirror effect and the condition to have an ion trapped in a dipole are also discussed. In the second part, simulations of the ion cloud dynamics with the NUAGE code [14] are shown in the framework of ThomX [15] $50 \mathrm{MeV}$ electron storage ring dedicated to $\mathrm{X}$ ray production via Compton backscattering. The equilibrium state modifications when using mitigation strategies are discussed (see Table I for the main parameters used in this article and Appendix A for the ThomX ring lattice).

\section{BEAM-ION INTERACTION AND THE MAGNETIC MIRROR EFFECT}

The beam-ion interaction can be described as a particular case of the beam-beam interaction in the "strong-weak" approximation [16]. The "strong-weak" model of the beambeam interaction considers that one beam, the "weak" beam, is not disturbing the propagation of the second beam, the "strong" beam, and only the effect of the "strong" beam on the "weak" beam is considered. In our case, the ion cloud is considered as the "weak" beam, a beam of low energy and intensity which will not disturb the electron beam. In this case, it is possible to express analytically the effect of an electron bunch, supposed Gaussian, on an ion. Since the transverse component of this interaction is well known and widely used whereas the longitudinal part is less usual, the longitudinal part will be more developed in this article.

\section{A. Transverse component of the beam-ion interaction}

To analyze the transverse part of the beam-ion interaction, one should start with the Coulombian interaction between an electron of the beam and an ion and deduce the velocity change that this ion will undergo during an infinitesimal time interval. Then sum this effect on all the electrons of a bunch supposed Gaussian in transverse dimensions and infinitely short in the longitudinal one. Talmann shows that these velocity kicks can be written in the form of a two-dimensional potential [16]. This potential expression can be used to express the ion velocity kicks as a function of the complex error function $w(z)$, Eq. (2), using the method of Bassetti and Erskine [17]. This makes it possible to obtain the transverse ion dynamics in terms of the transverse velocity kicks.

The transverse velocity kicks $\Delta v_{x}$ and $\Delta v_{y}$ are given by the Bassetti-Erskine formula, Eq. (1):

$$
\begin{gathered}
i \Delta v_{x}+\Delta v_{y}=\frac{-N K \sqrt{\pi}}{\sqrt{2\left(\sigma_{x}^{2}-\sigma_{y}^{2}\right)}}\left[w\left(\frac{x+i y}{\sqrt{2\left(\sigma_{x}^{2}-\sigma_{y}^{2}\right)}}\right)-e^{-\left[\frac{x^{2}}{2 \sigma_{x}^{2}}+\frac{y^{2}}{2 \sigma_{y}^{2}}\right]} w\left(\frac{x \frac{\sigma_{y}}{\sigma_{x}}+i y \frac{\sigma_{x}}{\sigma_{y}}}{\sqrt{2\left(\sigma_{x}^{2}-\sigma_{y}^{2}\right)}}\right)\right] . \\
w(z)=e^{-z^{2}}\left(1+\frac{2 i}{\sqrt{\pi}} \int_{0}^{z} e^{\tau^{2}} d \tau\right) .
\end{gathered}
$$

Where $\sigma_{x}$ and $\sigma_{y}$ are the horizontal and vertical electron beam size, $N$ is the number of electrons in a bunch, $K=\frac{2 r_{p} c^{2}}{v_{e} A} \approx \frac{2 r_{p} c}{A}, r_{p}$ is the classical proton radius, $v_{e}$ is the speed of the electrons, $c$ is the speed of light in vacuum, $A$ is the atomic mass of the ion, $x$ and $y$ are the transverse coordinates of the ion expressed relatively to the electron beam center of mass, and $w(z)$ is the complex error function defined in Eq. (2).
This formula gives the additional transverse velocity that an ion will gain when the electron bunch passes by the ion. This speed change depends on the longitudinal and transverse ion position. In particular, it depends on the variation of the beam transverse dimensions along the accelerator.

Contrary to most publications using the Bassetti-Erskine formula we get a minus sign in Eq. (1) since this expression is derived here in the case of two beams of opposite charge 
corresponding to the electron beam and the ion cloud. It is also important to note that in the Bassetti-Erskine formula the electric charge of the two beams does not appear clearly, it is hidden in $K=\frac{2 r_{p} c^{2}}{v_{e} A}=\frac{2 e^{2}}{4 \pi \epsilon_{0} A m_{p} v_{e}}$. Where $v_{e}$ is the velocity of the electrons, $e$ is the elementary charge, $\epsilon_{0}$ is the permittivity of free space and $m_{p}$ is the mass of the proton. The value of the charge of the two beams in the parameter $K$ should be adjusted to use this formula for multicharged ions.

At each bunch-ion interaction, ions feel a strong force toward the electron orbit. When an ion crosses the beam center in between two bunch-ion interactions, the following bunch-ion interaction will give a velocity kick of opposite sign from the previous ion velocity, bringing the ion back toward the electron beam orbit. The repetition of this effect explains the large transverse oscillations that ions undergo in the electron beam potential. As the Bassetti-Erskine formula is dependant on the beam size, it also explains why ions have different oscillation frequencies which depend on their longitudinal position in the machine. More details on the transverse beam-ion physics are available in $[8,18]$.

\section{B. Longitudinal component of the beam-ion interaction}

The "longitudinal" beam-ion interaction comes from the fact that the beam has a nonuniform transverse beam size. As shown in Fig. 1 the individual electron trajectories are not strictly parallel to the longitudinal axis $s$ when the beam envelope (of the electron beam) is varying. The force that one ion feels when an electron is passing by the ion is perpendicular to the electron trajectory. Consequently the velocity kick that the ion will feel has a longitudinal component in addition to the transverse one. The order of magnitude of longitudinal kicks is $1 \mathrm{~m} / \mathrm{s}$ compared to $100 \mathrm{~m} / \mathrm{s}$ for the transverse kicks but $\Delta v_{s}$ is not negligible. The effect on the longitudinal velocity accumulates over several hundreds of bunch-ion interactions and leads to ion accumulation points along the machine.

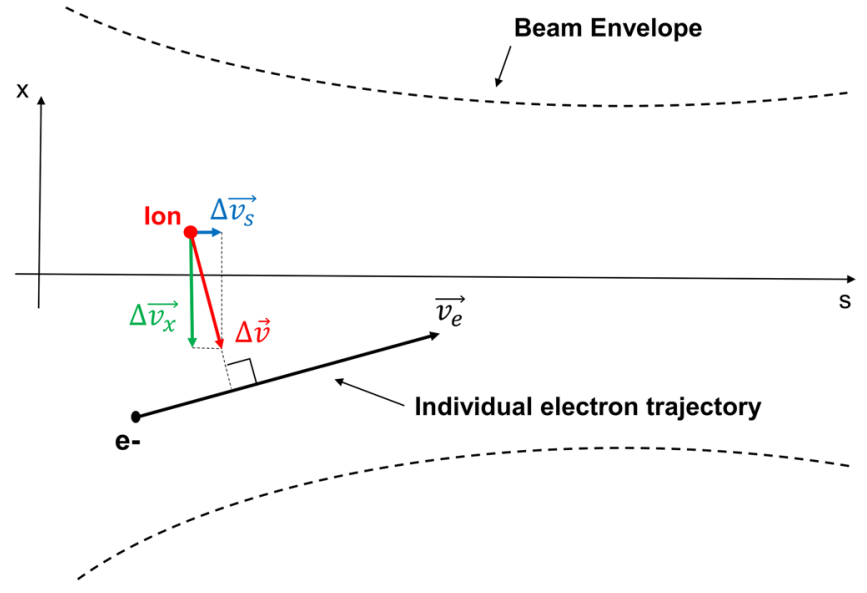

FIG. 1. The ion-beam interaction, the force felt by the ion is perpendicular to the local direction of the electron [13].
The derivation of the longitudinal velocity kick of the beam-ion interaction has been done by Sagan in [13]. The longitudinal velocity kick felt by an ion from a single electron with a trajectory quasiparallel to the longitudinal axis is obtained. Then this effect is summed over all the electrons of a Gaussian bunch to obtain Eq. (3):

$$
\Delta v_{s}=\left[-\alpha_{x} \epsilon_{x}+\left(\eta \sigma_{\epsilon}\right)\left(\eta^{\prime} \sigma_{\epsilon}\right)\right] \frac{\partial \Delta v_{x}}{\partial x}-\alpha_{y} \epsilon_{y} \frac{\partial \Delta v_{y}}{\partial y} .
$$

Equation (3) gives the longitudinal velocity kick $\Delta v_{s}$ as a function of the transverse kick derivative (see Appendix B), the optics of the machine and the beam parameters. Here $\alpha$, $\beta$ and $\gamma$ are the Twiss parameters, $\epsilon$ is the emittance, $\eta$ and $\eta^{\prime}$ are the dispersion function and its derivative and $\sigma_{\epsilon}=\frac{\sigma_{E}}{E_{0}}$ is the relative energy spread. All the terms of Eq. (3) depends on the longitudinal position $s$ except the emittances $\epsilon_{x, y}$ and the energy spread $\sigma_{\epsilon}$. The transverse kick derivatives $\frac{\partial \Delta v_{x}}{\partial x}$ and $\frac{\partial \Delta v_{y}}{\partial y}$ depend on both the longitudinal and transverse positions.

In Fig. 2 the color map of the longitudinal velocity kicks $\Delta v_{s}$ is drawn for the ThomX storage ring for $\mathrm{CO}^{+}$ions. Positive kicks (toward the right) are shown in red and negative kicks (toward the left) are shown in blue according to the color bar scale. The absolute value of the longitudinal velocity kick is maximum at the central orbit, i.e., when $x, y=0 \mathrm{~m}$, and decreases with a transverse displacement. Such a kick map is useful to obtain the ion accumulation points. For example, we see from Fig. 2 that in the region near of $s=4.5 \mathrm{~m}$, for $s<4.5 \mathrm{~m}, \Delta v_{s}>0 \mathrm{~m}$ and for $s>4.5 \mathrm{~m}, \Delta v_{s}<0 \mathrm{~m}$, so the ions will be pushed and trapped around this point. In fact, due to the longitudinal kicks on both sides of the accumulation point, the ions will oscillate longitudinally around this point. At $s=2.1 \mathrm{~m}$ and at $s=6.9 \mathrm{~m}$ there are two more accumulation points. The trapping region, the longitudinal width in which the ions are oscillating, at these two points is expected to be broader than at $s=4.5 \mathrm{~m}$ because of the uneven strength of the velocity kick creating these accumulation points.

An accumulation point is created when the sign of $\Delta v_{s}$ flips from positive to negative for increasing values of $s$. For a $\Delta v_{s}$ sign change from negative to positive, an expulsion zone is created. An expulsion zone is a longitudinal section in which ions created there will not stay: they will drift to an accumulation point or a free drifting section, a region where $\Delta v_{s}$ is small and not changing along s. In Fig. 2, two expulsion zones leading to weak accumulation points are located from $s=3 \mathrm{~m}$ to $s=3.4 \mathrm{~m}$, and from $s=5.5 \mathrm{~m}$ to $s=6 \mathrm{~m}$. Two more weak expulsion zones leading to free drifting sections are located from $s=0.8 \mathrm{~m}$ to $1.2 \mathrm{~m}$, and from $s=7.8 \mathrm{~m}$ to $s=8.2 \mathrm{~m}$. When $\Delta v_{s} \approx 0 \mathrm{~m} / \mathrm{s}$ there is no longitudinal kick in this section and ions drift with constant velocity in such a section. In Fig. 2, the free drifting section extends 


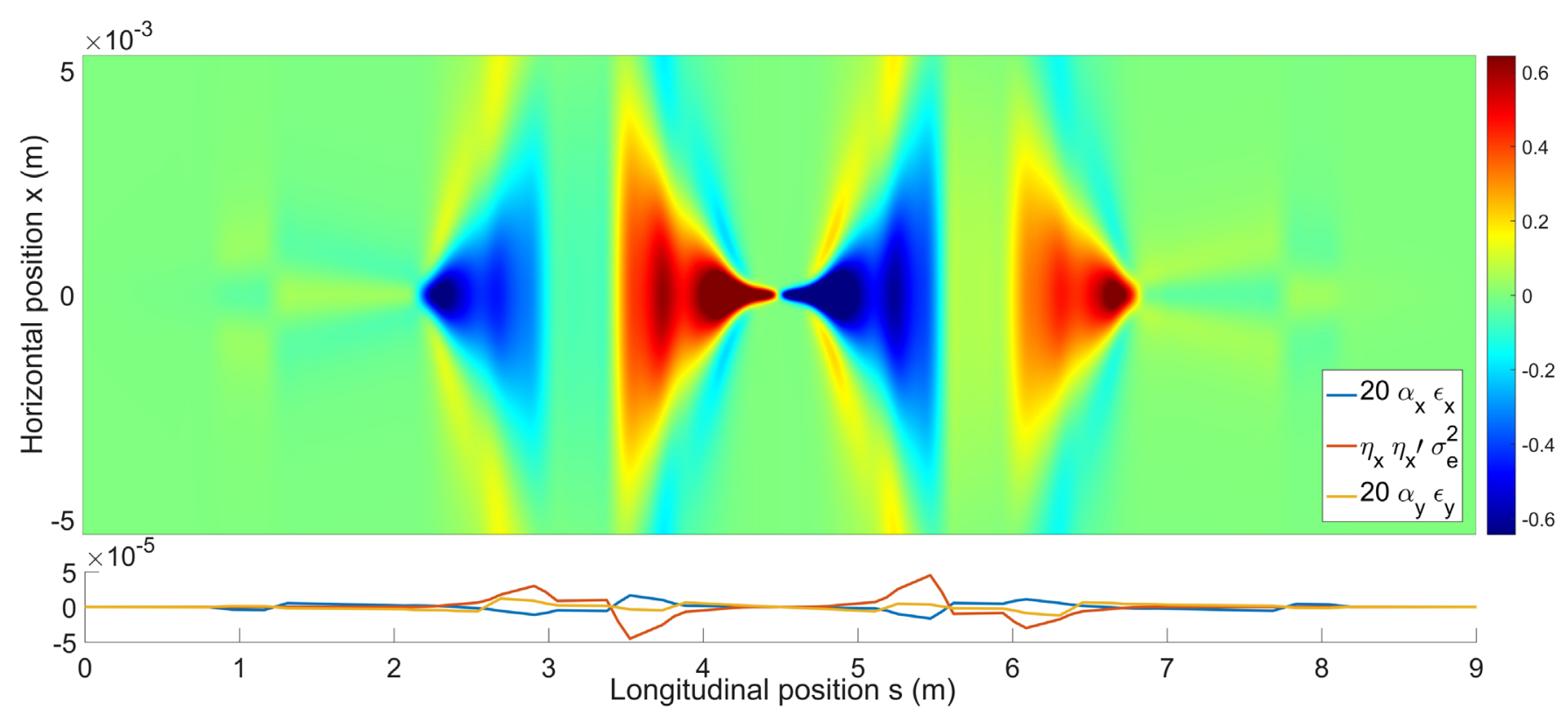

FIG. 2. Top: Color map of the longitudinal kicks $\Delta v_{s}$ vs the horizontal position $x$ and the longitudinal position $s$ along half of the ThomX ring for $\mathrm{CO}^{+}$ions $(A=28)$. Bottom: $20 \alpha_{x} \epsilon_{x}$ in blue, $\eta_{x} \eta_{x} \sigma_{\epsilon}{ }^{2}$ in orange and $20 \alpha_{y} \epsilon_{y}$ in yellow vs the longitudinal postion $s$ along half of the ThomX ring. The emittance terms of Eq. (3) have been multiplied by 20 in order to make their variations visible.

from $s=0 \mathrm{~m}$ to $s=0.8 \mathrm{~m}$ and from $s=8.2 \mathrm{~m}$ to $s=9 \mathrm{~m}$.

The sign of the longitudinal velocity kicks $\Delta v_{s}$ is given by the competition of the three terms of Eq. (3). For the ThomX storage ring, $\Delta v_{s}$ is dominated by the $\left(\eta \sigma_{\epsilon}\right)\left(\eta^{\prime} \sigma_{\epsilon}\right) \frac{\partial \Delta v_{x}}{\partial x}$ term as shown in the bottom plot of Fig. 2. The derivative of the transverse kick $\frac{\partial \Delta v_{x}}{\partial x}$ is negative in the central region. Then the accumulation points are located at dispersion minima, points where the dispersion derivative changes from a negative sign to a positive sign. ThomX case is a particular one because its energy spread is large compared to most high energy storage rings. For a typical synchrotron light source, the relative energy dispersion $\sigma_{\epsilon}$ would be lower and the $-\alpha_{x} \epsilon_{x} \frac{\partial \Delta v_{x}}{\partial x}$ term would either be the dominant one or in competition with the $\left(\eta \sigma_{\epsilon}\right)\left(\eta^{\prime} \sigma_{\epsilon}\right) \frac{\partial \Delta v_{x}}{\partial x}$ term. In the case of a ring dominated by the $-\alpha_{x} \epsilon_{x} \frac{\partial \Delta v_{x}}{\partial x}$ term, $\Delta v_{s} \approx \frac{1}{2} \frac{d \beta_{x}}{d s} \epsilon_{x} \frac{\partial \Delta v_{x}}{\partial x}$ and accumulation points would be located at the horizontal beta function $\beta_{x}$ minima.

Depending on the contributions of the three terms of Eq. (3), the ion accumulation points are located at different positions. If the energy spread $\sigma_{\epsilon}$ term is dominant, as in ThomX, then ions accumulate at the dispersion function $\eta$ minima. If one of the emittance $\epsilon_{x, y}$ terms is dominant, then ions accumulate at the beta function $\beta_{x, y}$ minima. If two, or all, terms are of the same order of magnitude then ions accumulate at longitudinal location $s=s_{0}$ such as $\Delta v_{s}\left(s_{0}\right)=0$ and $\frac{d \Delta v_{s}}{d s}\left(s_{0}\right)<0$. The localization of the longitudinal accumulation points gives the optimal location for a clearing electrode unless they are modified by magnetic fields. This will be discussed in the next section.

\section{Effect of dipoles and the magnetic mirror effect}

The motion of a particle of charge $q$ and mass $m$ in a uniform and constant magnetic field $\vec{B}$ is well known, it is the cyclotron motion. The charged particle performs an helical motion, superposition of a uniform motion along $\vec{B}$ and a circular motion in the plane normal to $\vec{B}$ with an angular velocity $\omega_{c}=\frac{q B}{m}$ and a radius $r_{c}=$ $\frac{v_{\perp}}{\omega_{c}}$ where $v_{\perp}$ is the velocity component which is perpendicular to the magnetic field. When an ion is inside a dipole magnet uniform magnetic field, it feels both the effect of the electron beam and the effect of the magnetic field.

In this section, we neglect the longitudinal effect of the beam-ion interaction and consider beam driven ion oscillations at angular velocities $\omega_{x, y}$ in the $x$ and $y$ directions. In that case, the vertical motion of the ion is unchanged by the dipole field and the ions still oscillate at pulsation $\omega_{y}$. The horizontal motion of the ions is the combination of the oscillations due to the beam at pulsation $\omega_{x}$ and due to the cyclotron motion at pulsation $\omega_{c}$. As usually $\omega_{x}>\omega_{c}$, the ions oscillate at pulsation $\omega_{x}$ with an amplitude modulation varying at pulsation $\omega_{c}$. The ion horizontal oscillations driven by the beam translates also into an ion longitudinal drift velocity $v_{d}$ given by Eq. (4) [12]: 


$$
v_{d}=\left(\omega_{c} x_{0}+v_{s_{0}}\right) \frac{\omega_{x}^{2}}{\omega_{c}^{2}+\omega_{x}^{2}}
$$

where $x_{0}$ is the initial horizontal position of the ion and $v_{s_{0}}$ is the initial longitudinal velocity. So the total longitudinal motion of an ion is the sum of this drift $v_{d}$ and the oscillation at angular velocity $\omega_{c}$ due to the cyclotron motion. Because of this longitudinal drift $v_{d}$, ions tend to drift away from the dipoles. In practice, ions are focused very near the beam axis. Therefore $x_{0}$ is small and the longitudinal drift velocity $v_{d}$ is limited.

In the fringe field of real dipole magnets, the magnetic field is nonuniform and changes with the longitudinal position $\vec{B}=\overrightarrow{B(s)}$. The first consequence of this is that, an ion moving in a dipole fringe field has a varying cyclotron angular velocity $\omega_{c}(s)$, cyclotron radius $r_{c}(s)$ and longitudinal drift velocity $v_{d}(s)$. A second consequence is that an ion coming from a magnetic field free region which enters the dipole can be reflected by the fringe field. This is called the "magnetic mirror" effect and the transmission or the reflection of the ion at the entrance of the dipole depends on initial conditions. In fact, it is the magnetic field gradient which is responsible for this effect and not the fringe field itself. It means that the effect is higher if the fringe fields are shorter as the gradient is higher. If the ion initial longitudinal velocity $v_{s_{0}}$ is high enough the ion will go through the fringe field else it will be reflected, the condition for the reflection [13] is

$$
v_{s_{0}} \leq\left|x_{0} \sqrt{\omega_{x} \Omega\left(B_{\max }\right)-\omega_{x}^{2}}\right| \approx\left|x_{0} \frac{\omega_{c}\left(B_{\max }\right)}{\sqrt{2}}\right| .
$$

Where $x_{0}$ is the initial horizontal position of the ion, $\Omega=\sqrt{\omega_{c}^{2}+\omega_{x}^{2}}$ and $\omega_{c}\left(B_{\max }\right)$ implies that $\omega_{c}$ should be computed for the highest value of the magnetic field $B(s)$. The approximate condition for reflection in Eq. (5) is valid for $\omega_{x} \gg \omega_{c}$ which is usually true.

Using this criteria in the ThomX case, and with $\omega_{x} \approx$ $2.6 \mathrm{MHz}$ and $\omega_{c} \approx 2.05 \mathrm{MHz}$, it takes a minimum longitudinal velocity $v_{s_{0}}$ of $1350 \mathrm{~m} / \mathrm{s}$ to go through a dipole field $B_{y}=0.597 \mathrm{~T}$ for an ion whose initial horizontal position is $x_{0}=1 \mathrm{~mm}$. The simulation shown in Fig. 3 agrees with this estimate. Figure 3 shows the evolution of the ion longitudinal position when going trough a ThomX dipole magnet for several initial longitudinal velocities. This simulation considers a $1 \mathrm{~m}$ long bend section with constant optics functions $\beta_{x}=10 \mathrm{~m}, \beta_{y}=9 \mathrm{~m}$ and $\eta=0 \mathrm{~m}$. The constant value of the dispersion function $\eta$ is not realistic but allows one to neglect the longitudinal effect of the beam-ion interaction to highlight the effect of the dipole magnet. The electron bunch has a repetition frequency of $16.7 \mathrm{MHz}$ which is the same as ThomX.

All the $100 \mathrm{CO}^{+}$ions start the simulation outside of the dipole magnetic field at the longitudinal position $s=$ $0.25 \mathrm{~m}$ with longitudinal velocities ranging linearly from

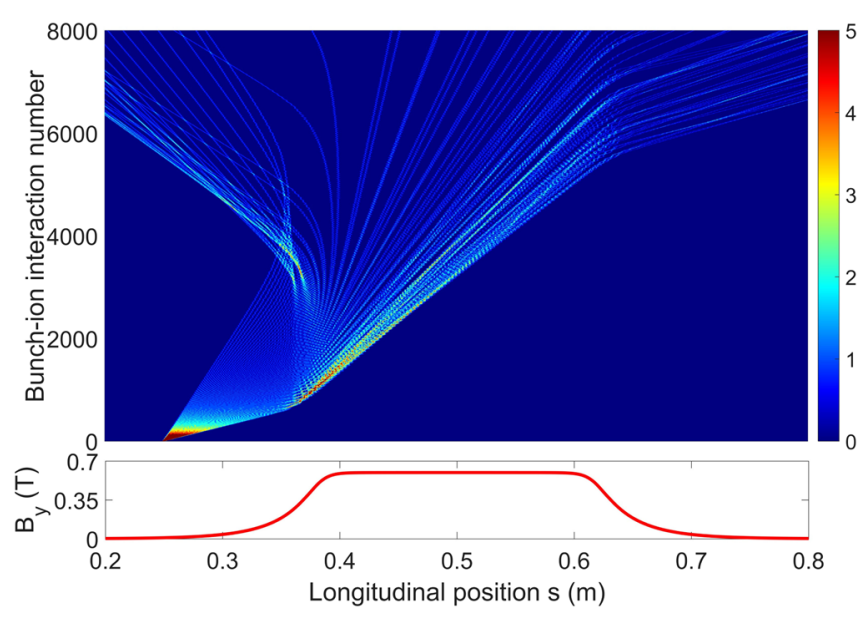

FIG. 3. Evolution of the ion longitudinal distribution when going trough a dipole magnet for various initial longitudinal velocities. The ion longitudinal distribution is shown in the top plot vs time (expressed in bunch-ion interactions) in ordinate and vs the longitudinal position s. The color scale shows the local density of ions (the number of ions per pixel). The bottom plot shows the vertical magnetic field $B_{y}$ as a function of the longitudinal position $s$. Results obtained using the NUAGE simulation code, see Sec. III A.

$v_{s}=500 \mathrm{~m} / \mathrm{s}$ to $3000 \mathrm{~m} / \mathrm{s}$. A fraction of the ions are reflected by the rising magnetic field when entering the dipole fringe field between $s=0.35 \mathrm{~m}$ and $0.4 \mathrm{~m}$. The minimum initial velocity of all the transmitted ions is $v_{s_{0}} \approx$ $1300 \mathrm{~m} / \mathrm{s}$ which is close to the value computed using Eq. (5), $v_{s_{0}}=1350 \mathrm{~m} / \mathrm{s}$. The longitudinal velocity increase of the transmitted ions while inside the dipole, from $s=0.4 \mathrm{~m}$ to $s=0.6 \mathrm{~m}$, corresponds to the longitudinal drift velocity $v_{d}$, Eq. (4).

There are at least two cases where ions can be trapped by the magnetic mirror effect. The first possibility, case I, is to have ions with low longitudinal velocities between two dipoles. Then the ions are reflected back and forth from one dipole fringe field to the other and accumulate in the straight section between the two dipoles. Another possibility, case II, is to have an ion accumulation point due to the beam-ion interaction at a dipole location. If the longitudinal kicks from the beam-ion interaction are not strong enough for the ions to go through the dipole fringe field, then ions oscillate in the dipole fringe field. In that case, ions gather around the longitudinal position $s_{0}$ where the longitudinal beam-ion kick is compensated by the longitudinal drift speed from the dipole: $\left|\Delta v_{s}\left(s_{0}, x_{0}, y_{0}\right)\right|=\left|v_{d}\left(s_{0}, x_{0}\right)\right|$.

\section{SIMULATION OF THE ION CLOUD DYNAMICS IN THOMX STORAGE RING}

Simulations are needed to fully understand the dynamics of the ion cloud in an accelerator. As explained in Sec. II, ions are strongly impacted by the beam-ion interaction and by magnetic fields. In order to get the equilibrium 
distribution of the ions both effects should be simulated together.

In the first part of this section, the NUAGE simulation code [14] is presented. In the second part, simulation results are shown for the ThomX $50 \mathrm{MeV}$ storage ring without mitigation techniques. In the third and fourth parts, the effect of clearing electrodes and clearing gaps are discussed.

\section{A. The NUAGE simulation code}

NUAGE is a data parallel Matlab program which tracks ion clouds in electron storage rings. The beam-ion interaction model is based on the Bassetti-Erskine formula for the transverse ion motion and on the Sagan formula for the longitudinal motion as explained in Sec. II. The ion tracking in magnetic fields (and in curved sections) is done by numerically solving the differential equation of motion in magnetic fields in a curvilinear frame. The effect of DC clearing electrodes can be taken into account by using the 3D electric field, computed with an electrostatic solver, used as an input to the program.

In NUAGE, all the ions considered are created at the start of the simulation. Usually their positions follow the Gaussian beam distribution in the transverse plane and are uniformly distributed along the beam in the longitudinal direction. A Maxwell-Boltzmann distribution at room temperature is used for their speeds. The NUAGE code then tracks the ion cloud for a fixed duration defined by the user.

As the ion generation rate is not directly dealt with in the program, it is important to take it into account in the analysis. The tracking with NUAGE from an initial distribution to a final one allows one to extract the clearing coefficient $\epsilon(t)$ defined by Eq. (6). The clearing coefficient is the ratio of the number of tracked ions $N_{i}(t)$ at time $t$, which were not cleared during the tracking, over the number of tracked ion at the start of the simulation $N_{i 0}$. Using the clearing coefficients and the known ion production rates, it is possible to get global values like the neutralization factor $\eta(t)=\frac{N_{i}(t)}{N_{e}}$ defined as the ratio of trapped charges to beam charges.

$$
\epsilon(t)=\frac{N_{i}(t)}{N_{i 0}} .
$$

Different benchmarks have been done to test NUAGE code: the trapping critical mass [1], the ion oscillation frequencies [6] and the transverse equilibrium distribution [6] simulated with NUAGE have been found to match the theoretical predictions. A benchmark of the magnetic mirror effect is presented in Sec. II C. Several approximations and hypotheses are made during the tracking and the analysis. Among them, the beam-ion model of Sec. II is adopted. The influence of a nonuniform vacuum profile is neglected, i.e., a uniform vacuum profile is assumed. The vacuum profile should have a relatively small impact on the ion longitudinal equilibrium distribution as the longitudinal beam-ion force is the main driver of the equilibrium state. Also, the equilibrium state obtained by NUAGE does not take into account the constant generation of new ions which drift from their generation position to accumulation points. In reality there is a constant flow of new ions which slightly modify the equilibrium distribution [2]. The ion space charge is not taken into account, but in most cases this effect is expected to be small. In [10], the ion cloud effect is simulated with the ion cloud space charge to compute the ion oscillation frequencies and compared with the results given by the linear theory (without space charge). The good agreement found is a good indication to confirm that the space charge effect is small for ion clouds. The small difference remaining between the result found and the linear theory can be explained by the nonlinearities. More details on the NUAGE code and the analysis method are provided in [14].

\section{B. Propagation toward the equilibrium distribution}

In this section, the dynamics of an ion cloud is simulated from its generation to its equilibrium distribution. At the start of the simulation, all ions are distributed uniformly along the ring longitudinal position. Figure 4 shows the evolution of the $\mathrm{CO}^{+}$ion longitudinal distribution along the longitudinal position $s$ vs the number of bunch-ion interactions. The color scale shows the local ion density (the number of ions per pixel) at a given longitudinal position and at a given time. A red area indicates a high density of ions whereas a dark blue one indicates the absence of ions. The ion cloud is tracked for 50000 bunch-ion interactions, and reach its equilibrium state in approximately 25000 interactions. The bottom plot of Fig. 4 is a synopsis of the ThomX storage ring elements taken into account for the simulation. Ions are tracked in the dipole and quadrupole magnets. Dipoles are modeled with their longitudinal fringe fields.

As it can be seen in Fig. 4, ions drift from their initial position to accumulation points. The accumulation points expected from the longitudinal beam-ion interaction in Sec. II B at $s=2.1 \mathrm{~m}, s=4.5 \mathrm{~m}$ and $s=6.9 \mathrm{~m}$ are found in the simulation. At $s=4.5 \mathrm{~m}$, ions seem tightly focused longitudinally by the beam-ion force, whereas at $s=2.1 \mathrm{~m}$ and $s=6.9 \mathrm{~m}$ ions are less focused and drift near these points. The reason for this is the uneven strength of the longitudinal kicks, as explained in Sec. II B, and the presence of dipole magnets at $s=2.1 \mathrm{~m}$ and at $s=6.9 \mathrm{~m}$, near the accumulation points, which compete with the beam-ion force. At $s=4.5 \mathrm{~m}$, there are two dipoles, one on each side of the accumulation region but not directly superposed to the accumulation point.

Near the accumulation point located at $s=2.1 \mathrm{~m}$, the ions stay mostly on the left side of the accumulation point as the kicks on the right side are much stronger as it can be seen in Fig. 2. Some ions are trapped on the right side of the accumulation point: the beam kicks do not give them 


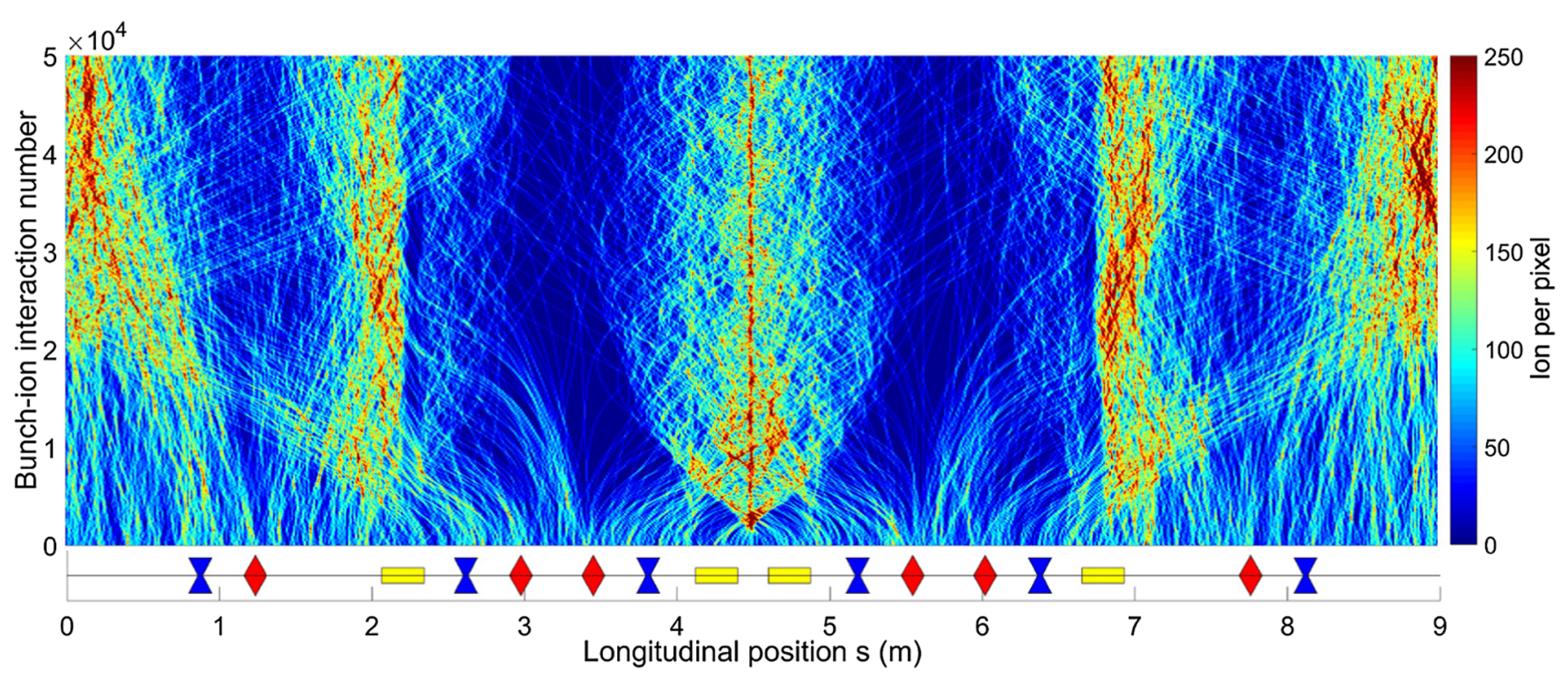

FIG. 4. Tracking of a $\mathrm{CO}^{+}$ion cloud during 50000 bunch-ion interactions, starting from an uniform distribution in the longitudinal dimension. The ion density is plotted vs the time (expressed in bunch-ion interactions) in ordinate and vs the longitudinal position for half of the ThomX storage ring in abscissa. The number of ions at the start of the simulation is $N_{i 0}=10^{5}$. The bottom plot is a synopsis of the ThomX storage ring elements taken into account for the simulation. Focusing quadrupoles are represented in blue, defocusing quadrupoles are represented in red and dipoles in yellow.

enough velocity to enter the dipole so they are reflected backward and oscillate in the fringe field region (case II). Case I trapping, ions with low velocity oscillating between two dipoles, occur at $s=4.5 \mathrm{~m}$. The case II trapping also happens at $s=4.1 \mathrm{~m}, s=4.9 \mathrm{~m}$ and $s=6.6 \mathrm{~m}$, this effect is more visible with clearing electrodes and is highlighted by red dotted arrows in Fig. 6 .

The empty regions in Fig. 4, from $s=3 \mathrm{~m}$ to $s=3.4 \mathrm{~m}$ and from $s=5.5 \mathrm{~m}$ to $s=6 \mathrm{~m}$, correspond to expulsion zones. In the free drifting sections, from $s=0 \mathrm{~m}$ to $s=0.8 \mathrm{~m}$ and from $s=8.2 \mathrm{~m}$ to $9 \mathrm{~m}$, although there is no kick due to the beam-ion force many ions gather there. Ions are slowly pushed to these regions by the weak expulsion zones from $s=0.8 \mathrm{~m}$ to $s=1.2 \mathrm{~m}$ and from $s=7.8 \mathrm{~m}$ to $s=8.2 \mathrm{~m}$.

This simulation illustrates the very fast propagation of the ions toward their accumulation position. Dipole magnets modify the equilibrium state with ion reflections on fringe fields. In this case the effect of quadrupoles is very small and bring only small modifications to the ion trajectories. In the transverse plane the ions quickly, in about 1000 bunch-ion interactions, converge toward an equilibrium distribution which is very peaked at the electron beam center [6]. As a result, most of the ions are inside the electron beam, very near the axis where the quadrupole magnetic field is low.

\section{Clearing electrodes}

Setting up clearing electrodes is an usual way of mitigating conventional ion trapping (CIT) in small electron storage rings and ERLs [8,19]. Usually clearing electrodes are polarized plates placed into the vacuum chamber to provide a transverse electric field which collects the ions on the electrodes. Because of the relatively small constant potential $\mathrm{U}$ applied to such electrodes, on the order of one $\mathrm{kV}$, clearing electrodes do not affect the electron dynamics. They are not generally used in large rings as covering a large machine with such electrodes is costly. As any accelerator component, clearing electrodes increase the total impedance of the accelerator, so a careful design of the electrodes is needed.

In order to achieve a good ion clearing with electrodes, electrode positioning is crucial and a study of the accumulation regions should be done before choosing electrode positions. Because dipoles can add additional trapping and are often near the accumulation points (because of the dispersion change at dipole positions), setting the clearing electrodes near or inside dipole magnets is generally effective.

Depending on the design of the electrodes, it is possible to generate both a transverse and a longitudinal electric field and not only the usual transverse field. In that case, using a negative polarity the longitudinal field is such that it creates an artificial longitudinal accumulation point at the electrode position (a positive polarity would create an expulsion point). This longitudinal field helps as it will bring more ions to be cleared by the transverse field. A longitudinal field is created by the DC voltage difference between the electrodes and the vacuum chamber, which is grounded. 

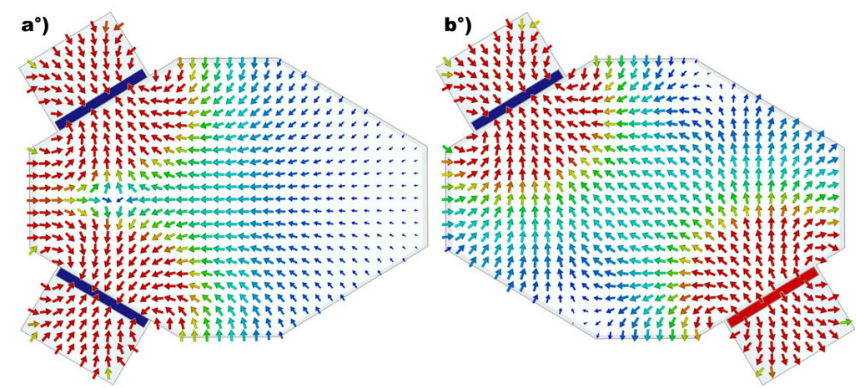

FIG. 5. Transverse electric field created by a clearing electrode composed of two buttons. The color scale for the arrows is linear from red, $E=20 \mathrm{kV} / \mathrm{m}$, to blue, $E=0 \mathrm{kV} / \mathrm{m}$. Electrode (a): configuration $-\backslash-$, the two buttons are negatively polarized, $U=-0.5 \mathrm{kV}$. Electrode (b): configuration $-\backslash+$ the upper button is negatively polarized, $U=-0.5 \mathrm{kV}$, and the lower button is positively polarized, $U=0.5 \mathrm{kV}$.

In ThomX, several different designs of clearing electrodes with multiple buttons are used, they are in fact similar to the beam position monitors designed by Soleil [20]. In the case of an electrode composed of several buttons as in Fig. 5, the possibility to produce a longitudinal electric field depends on the symmetry. In the case of electrode (a), the zero of the transverse field is not at the vacuum chamber center as the buttons are not symmetric with respect to the cavity center. In the other case [electrode (b)], the buttons are symmetric with respect to the vacuum chamber center so to have both buttons with negative polarity would not be efficient as the transverse field would be zero at the cavity centrer where the ions are. So for electrode (b), the two buttons have an opposite polarity which produces a transverse field but no longitudinal field along the beam axis. It would be possible to get both fields in the cavity center using only one button polarized negatively but the maximum amplitude of the field at the cavity center is much lower in that configuration.

Table II summarizes the simulation study done using NUAGE for 10000 bunch-ion interactions for different polarization configurations of the electrode buttons (a) and (b). A $20 \mathrm{~cm}$ straight section with constant optic functions $\beta_{x}=10 \mathrm{~m}, \beta_{y}=9 \mathrm{~m}$ and $\eta=0 \mathrm{~m}$ is used for the test, and the electron beam has a repetition frequency of 16.7 $\mathrm{MHz}$ which is the same one as ThomX. For a type (a) electrode, only $18 \%$ of the initial ions remain after 10 000 bunch-ion interactions for configuration $-\backslash-$. The longitudinal electric field produced by the $-\backslash-$ configuration allows $15 \%$ more clearing compared to the equivalent configuration, $+\backslash-$, without longitudinal field.

Figure 6 shows the tracking of a $\mathrm{CO}^{+}$ion cloud in half the ThomX storage ring using clearing electrodes. The simulation shown here is similar to the one shown in Fig. 4 but with five electrodes clearing the ions. Three type (a) electrodes are located at $s=3.1 \mathrm{~m}, s=4.5 \mathrm{~m}$ and $s=5.9 \mathrm{~m}$, two type (b) electrodes are located at $s=0.7 \mathrm{~m}$ and $s=8.3 \mathrm{~m}$. The electrode at $s=4.5 \mathrm{~m}$ is placed at the
TABLE II. Percentage of remaining ions after 10000 bunch-ion interactions in a $20 \mathrm{~cm}$ straight section. The configuration indicates the electrode polarization, the first sign corresponds to the upper electrode and the second one for the lower electrode. Respectively, + stands for $U=0.5 \mathrm{kV}$,- for $U=-0.5 \mathrm{kV}$ and 0 for $U=0 \mathrm{~V}$. From NUAGE simulations with $\mathrm{CO}^{+}$ions and $N_{i 0}=16000$.

\begin{tabular}{lcc}
\hline \hline Type & Configuration & Percentage of remaining ions \\
\hline (a) & $-\backslash-$ & $18 \%$ \\
(a) & $+\backslash-$ & $33 \%$ \\
(a) & $+\backslash+$ & $78 \%$ \\
(b) & $-\backslash-$ & $29 \%$ \\
(b) & $+\backslash-$ & $31 \%$ \\
(b) & $+\backslash+$ & $94 \%$ \\
(b) & $-\backslash 0$ & $31 \%$ \\
\hline \hline
\end{tabular}

main accumulation point and allows the clearing of most of the ions there. The two electrodes of type (b) are located near the exit of the expulsion zones, i.e., at $s=0.8 \mathrm{~m}$ and $s=8.2 \mathrm{~m}$. This helps to isolate the free drifting regions and to prevent ions from accumulating too much in this zone. The two electrodes at $s=3.1 \mathrm{~m}$ and $s=5.9 \mathrm{~m}$ are not very effective but decrease to some extent the number of ions trapped near the weak accumulation points at $s=2 \mathrm{~m}$ and $s=7 \mathrm{~m}$. The ion reflection by the dipole fringe fields is visible at $s=2.3 \mathrm{~m}, s=4.1 \mathrm{~m}, s=4.9 \mathrm{~m}$ and $s=6.7 \mathrm{~m}$.

As shown by this simulation, clearing electrodes should allow to clear an important fraction of the ions. There are still two uncleared accumulation regions at $s=2 \mathrm{~m}$ and $s=7 \mathrm{~m}$ but due to the overcrowding of the machine it was not possible to install clearing electrodes there. Still, electrodes should reduce the number of ions accumulated by a factor 3 , see Fig. 9 .

\section{Clearing gaps}

Clearing gaps are also a widely used mitigation technique to fight ion trapping. For machines working with multiple bunches it consists in having empty rf buckets (without bunches) in a given pattern. The absence of the beam focusing force on the ions perturb the ion transverse oscillations and leads to ion clearing. A long gap in the bunch train can be used to avoid conventional ion trapping while the use of multibunch train beam fillings has been shown to be very effective to counteract the fast ion instability [6].

For a storage ring working with only one bunch, as ThomX, the concept of clearing gap is different. In ThomX a bunch is stored for $20 \mathrm{~ms}$, and used to produce $\mathrm{X}$ rays via Compton scattering, then it is dumped while a new bunch is injected. It is possible to delay by some time the injection of the new bunch to make the ion clearing more effective. In that case, the clearing gap is the duration between extraction of the used bunch and the injection of a new one. The 


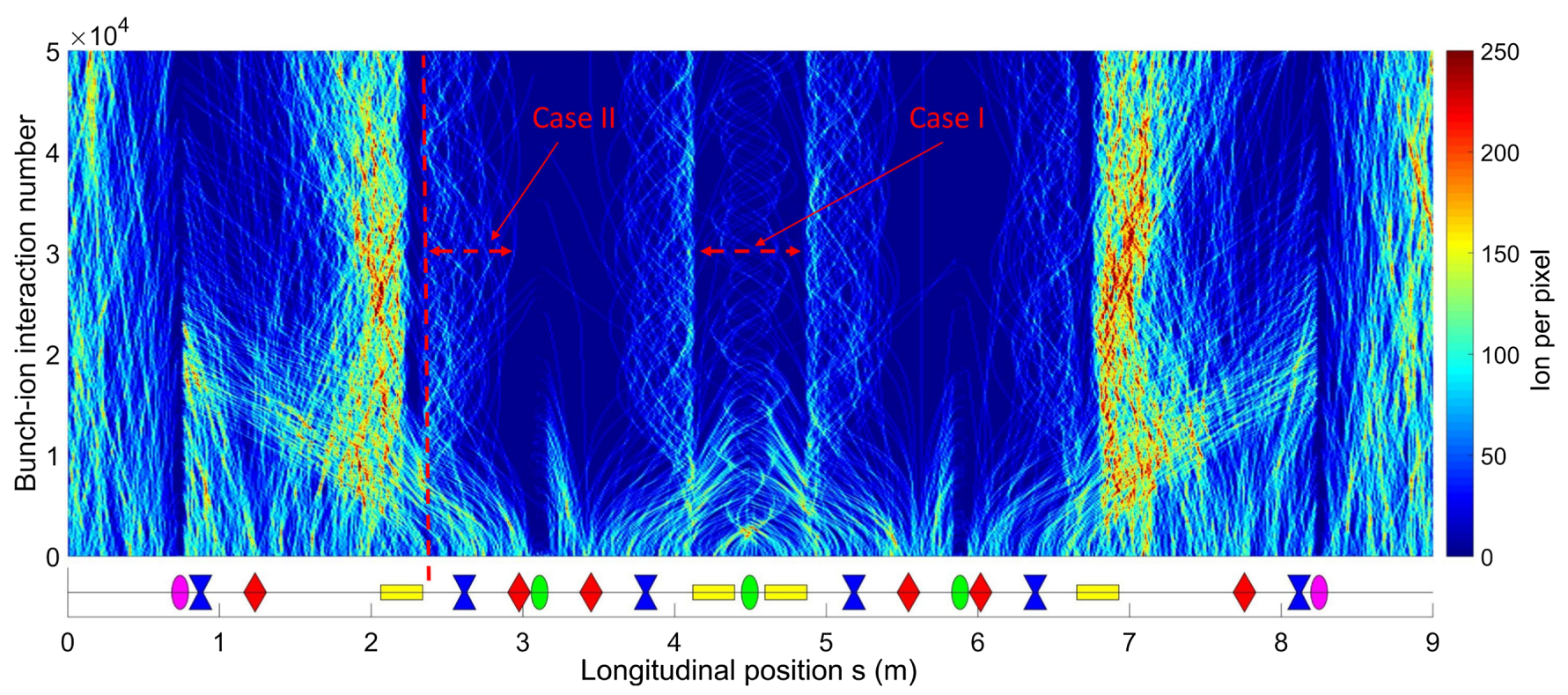

FIG. 6. Tracking of a $\mathrm{CO}^{+}$ion cloud during 50000 bunch-ion interactions with clearing electrodes on. The bottom plot is a synopsis of ThomX storage ring elements taken into account for the simulation. Number of ions at the start of the simulation $N_{i 0}=10^{5}$. Focusing quadrupoles are represented in blue, defocusing quadrupoles are represented in red, dipoles in yellow. Clearing electrodes of type (a) in configuration $-\backslash-$ are shown in green and electrodes of type (b) in configuration $+\backslash-$ in magenta. Red dotted arrows highlight the effect of ion trapping of case I and II due to the dipoles.

physical effect of such a clearing gap on the ions is the same as the one implemented in multibunch machines.

In Sec. III B the ion dynamics was described in the case of ions generated uniformly along the orbit. The equilibrium state obtained was modified in Sec. III C by the effect of the clearing electrodes. The ion cloud in its equilibrium state, reached after 350000 bunch-ion interactions (approximately $20 \mathrm{~ms}$ ) with clearing electrodes, is taken as the input for the simulation shown in Fig. 7. It shows the evolution of the vertical distribution of all the ions in the accelerator (whatever their longitudinal position) during the clearing gap of $4 \mu \mathrm{s}$ (66 missed bunch-ion interactions). At the start of the clearing gap, all the ions are tightly packed by the beam-ion interaction within $300 \mu \mathrm{m}$ of the beam mean orbit. During the gap the ions are free to escape from the beam envelope as there is no beam-ion interaction.

Since the gap duration here is short, less than $1 \%$ of the $\mathrm{CO}^{+}$ions are cleared during this gap. For lighter ions like $\mathrm{H}_{2}^{+}$, the same study shows that around $12 \%$ of the ions will be cleared during a $4 \mu \mathrm{s}$ gap. But even if the ions are not cleared during the gap, the ion vertical positions are mixed up, due to the natural divergence of the ion cloud, which brings an increased clearing efficiency when the beam-ion interaction begins anew. This effect is presented in Fig. 8, which shows the simulation of the ion cloud evolution after the clearing gap. The input ions are the ions with mixed up positions after the gap. The change in the ion transverse position, because of the gap, leads to an increased longitudinal velocity which help ion clearing. Ions which were trapped in the weak accumulation points, at $s=2.1 \mathrm{~m}$ and $s=6.9 \mathrm{~m}$, escape the accumulation regions because of the gap. The spreading of the positions also impacts the ions trapped in dipole fringe fields and helps to clear them.

The clearing gaps allow one to clear the ions all along the ring and are effective to clear the accumulation regions where there are no electrodes. Even a short gap during which ions are not cleared but their positions are spread is

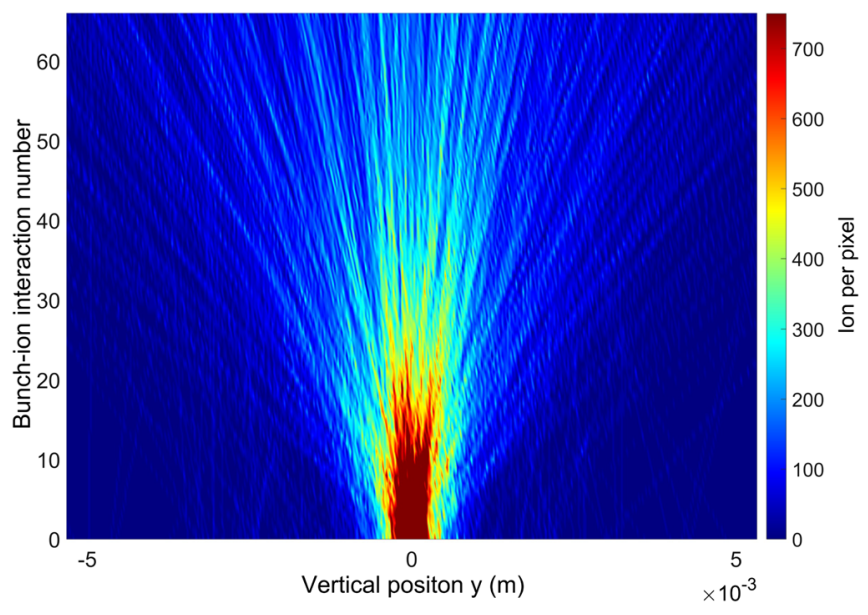

FIG. 7. Tracking of a $\mathrm{CO}^{+}$ion cloud during the clearing gap. The ion vertical distribution is plotted vs time (expressed in number of bunch-ion interactions) and the vertical position $y$. The beam-ion interaction is switched off but the effect of magnets and clearing electrodes is present. Number of ions at the start of the simulation $N_{i 0}=35000$. 


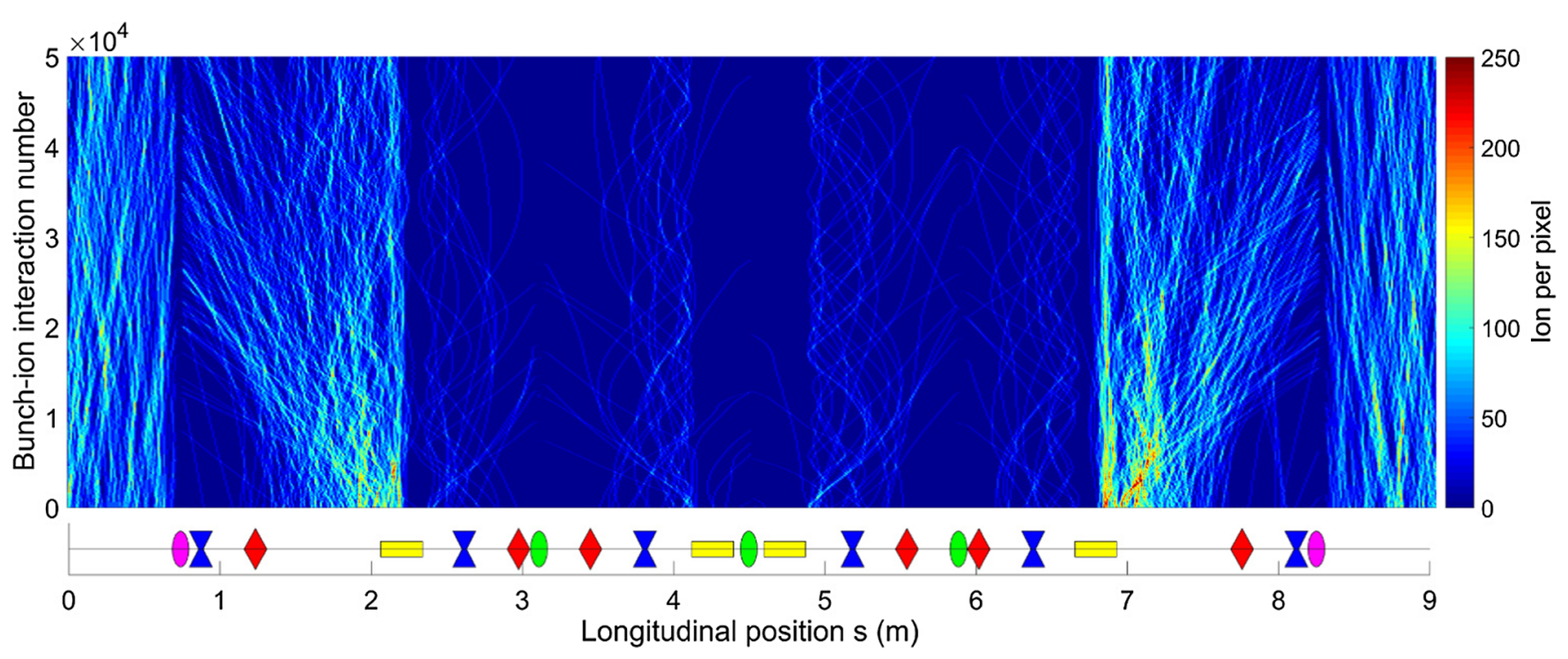

FIG. 8. Tracking of a $\mathrm{CO}^{+}$ion cloud during 50000 bunch-ion interactions just after a clearing gap with clearing electrodes on. The ion number at the start of the simulation is $N_{i 0}=35000$.

useful as the clearing efficiency is increased after the gap. In Fig. 9 the clearing coefficient $\epsilon(t)$, Eq. (6), is plotted vs time for several simulations. After 350000 bunch-ion interactions, $90 \%$ of the initial ions are still trapped if there are no clearing electrodes but only $30 \%$ of the ions remain if the clearing electrodes are switched on. It is interesting to note that in both cases the clearing is efficient at the beginning of the simulation, then it reaches a plateau

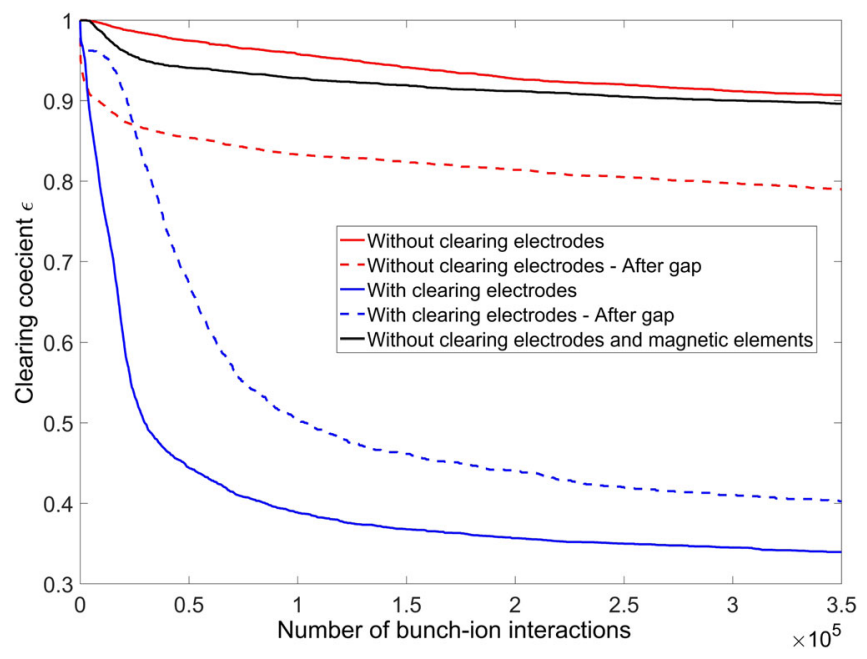

FIG. 9. Clearing coefficient $\epsilon$ vs the time, expressed in bunchion interactions, for different simulations for $\mathrm{CO}^{+}$ions. Blue lines correspond to simulations with clearing electrodes and red lines without. Plain lines correspond to simulations of the ion cloud generated with an uniform distribution. Dashed lines correspond to simulations of the ion cloud after a clearing gap. The black plain line corresponds to a simulation without clearing electrodes and magnetic elements. where the ion number decreases very slowly. Note that, after the gap, a new simulation begins so the clearing coefficient $\epsilon$ is normalized by the amount of ions at the start of the new simulation, which is the number of ions still present after the gap, so $\epsilon=1$. After some time only the ions which have the most stable positions and are away from clearing electrodes remain, which is why a plateau is reached. The use of a clearing gap allows one to leave the plateau and once again to clear ions as it spreads ion positions. The difference between the clearing coefficient with and without clearing electrodes is the consequence of the increased longitudinal velocity, due to the clearing gap, which bring ions to the clearing electrode locations.

The difference between the solid red line and the black line shows the role of magnetic elements in the increased ion trapping. The effect is relatively small for $\mathrm{CO}^{+}$ions, about a $3 \%$ increase in ion trapped, but is not a negligible quantity for $\mathrm{H}_{2}^{+}$ions where the increase reach $25 \%$. The ion trapping is stronger for $\mathrm{H}_{2}^{+}$ions because the minimum longitudinal velocity to go through a dipole fringe field scale as $\frac{1}{A^{2}}$, Eq. (5).

\section{E. Neutralization factor}

From the previous simulations and from the values of the clearing coefficients $\epsilon$ it is possible to obtain the neutralization factor $\eta(t)=\frac{N_{i}(t)}{N_{e}}$. The neutralization factor $\eta$ is the ratio of the number of trapped ions $N_{i}$ to the number of electrons in a bunch $N_{e}$. This value is directly related to the effects of the ions on the electron beam properties such as tune shift and tune spread [8].

The ion number evolution can be computed by solving differential equations combining the different ionization 
processes and the clearing coefficients. It allows one to deal with a reasonable number of particles and save computation time compared to the case where a realistic number of ions is simulated. The clearing coefficient $\epsilon$ can also be seen as the probability of survival of an ion when it is generated in a given distribution. Ions produced by beam ionization are distributed uniformly along the accelerator, so their probability to survive after a time $t$ is given by the solid blue line of Fig. 9 (case with electrode on). The ions which are not cleared propagate quickly towards an equilibrium distribution. Before a clearing gap, all the ions are considered as in the equilibrium distribution. After a clearing gap, the probability of an ion alive before the gap to survive after a time $t$ is given by the dashed blue line of Fig. 9 (case with electrodes on). In this analysis, ions have a "memory" which translates the fact that ions in different distributions behave differently and do not have the same survival probability.

In ThomX, the ion production rate due to beam ionization is $N_{\text {new }}^{\mathrm{CO}^{+}} \approx 9.3 \times 10^{6} \mathrm{ion} / \mathrm{m} / \mathrm{s}$ for $\mathrm{CO}^{+}$ions and $N_{\text {new }}^{\mathrm{H}_{2}^{+}} \approx 1.6 \times 10^{7} \mathrm{ion} / \mathrm{m} / \mathrm{s}$ for $\mathrm{H}_{2}^{+}$ions. With clearing electrodes off and no clearing gaps, a simple calculation gives a neutralization time, i.e., the time it takes to reach $\eta=1$, of $14 \mathrm{~s}$. Figure 10 is obtained by using the clearing coefficients simulated for $\mathrm{CO}^{+}$and $\mathrm{H}_{2}^{+}$ions and the cross sections of the different processes which generate ions from residual molecules [21]. It shows the evolution of the neutralization factor $\eta$ for several $20 \mathrm{~ms}$ cycles of beam-ion interaction each one followed by a $4 \mu$ s clearing gap.

Between $t=0 \mathrm{~ms}$ and $t=20 \mathrm{~ms}$, the neutralization factor $\eta$ increases as ions are produced by ionization.

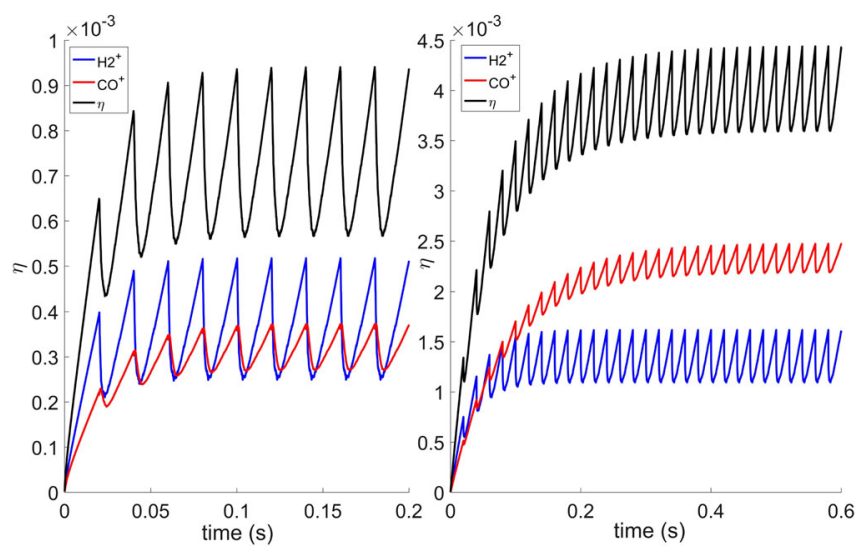

FIG. 10. The neutralization factor $\eta$ vs time with clearing electrodes on the left and without clearing electrodes on the right. The contribution of $\mathrm{CO}^{+}$ions to the neutralization factor is in red, the contribution of $\mathrm{H}_{2}^{+}$ions is in blue and the full neutralization factor is in black. The left plot shows 10 cycles of $20 \mathrm{~ms}$ of beamion interaction followed by a $4 \mu$ s clearing gaps while the right plot shows 30 cycles. Initial residual gas mix: total pressure $P_{t}=3 \times 10^{-10}$ mbar with $90 \%$ of $\mathrm{H}_{2}$ and $10 \%$ of $\mathrm{CO}$.
After $t=20 \mathrm{~ms}$, the neutralization factor $\eta$ drops because the gap increase ion clearing. The effect of the gap is partly balanced by the new ions produced so that after two cycles at $t=40 \mathrm{~ms}$ the total number of ions has increased. After some cycles, a dynamic equilibrium is reached when the new ions compensate the ions cleared by the gap. The use of clearing electrodes allows one to reach this state in fewer cycles than without electrodes. It also decreases the maximum value of the neutralization factor from $\eta \approx 4.5 \times 10^{-3}$ to $\eta \approx 1 \times 10^{-3}$.

\section{CONCLUSION}

Through simulations, the importance of the longitudinal motion of ions within electron accelerators has been investigated. The longitudinal motion is driven not only by the beam-ion interaction but also by the magnetic elements. Simulations of these effects allow one to obtain the ion equilibrium longitudinal distribution. The knowledge of the accumulation points along the accelerator is crucial to properly position clearing electrodes in order to maximize the clearing efficiency. Depending on the design of the clearing electrodes, it is possible to create artificial longitudinal accumulation points at the electrode location in order to optimize the clearing. The mechanism behind clearing gaps has been highlighted by simulations. This study of the ThomX storage ring allowed us to find a design that minimizes the ion effects and lowers the neutralization factor down to $\eta \approx 10^{-3}$. This value is well below the instability threshold of the classical ion trapping instability (CIT), around $10^{-1}$ or more [22]. The estimated ioninduced tune shift (and tune spread) is around $1 \times 10^{-4}$ with clearing electrodes and around $8 \times 10^{-4}$ without clearing electrodes [1].

\section{ACKNOWLEDGMENTS}

The authors would like to thank David Sagan for his kind reply to our questions about the longitudinal ion dynamics and the derivation of his equation. The authors wish to thank Jacques Haissinski and Marica Biagini for proofreading this article. Alessandro Variola, Nicolas Hubert, Theo Demma and Ryutaro Nagaoka are also acknowledged for fruitful discussions. Engineering office and mechanical department from LAL are thanked for exchanges concerning the clearing electrodes and the insertion of the devices in the vacuum tubes. This work is supported by ANR-10-EQPX-51, by grants from Région Ile-de-France and IN2P3.

\section{APPENDIX A: THOMX LATTICE}

Figure 11 shows the nominal optics of the ThomX storage ring. The bottom plot of Fig. 11 is a synopsis of the 


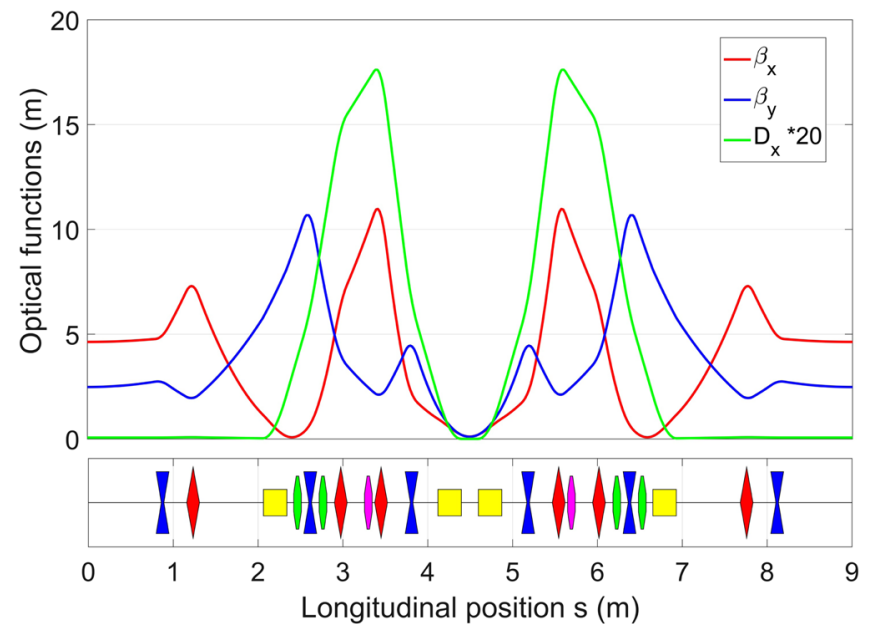

FIG. 11. Optics functions for half of the ThomX storage ring lattice. The horizontal beta function $\beta_{x}$ is plotted in red, the vertical beta function $\beta_{y}$ in blue and the horizontal dispersion times 20 is plotted in green. The bottom plot is a synopsis of the ThomX elements.

ThomX elements. Focusing quadrupoles are represented in blue, defocusing quadrupoles are represented in red and dipoles in yellow. Focusing sextupoles are shown in green and defocusing sextupoles in pink.

\section{APPENDIX B: TRANSVERSE BEAM-ION KICK DERIVATIVE}

To compute the longitudinal part of the beam-ion interaction $\Delta v_{s}$ from Eq. (3), the partial derivatives $\frac{\partial \Delta v_{x}}{\partial x}$ and $\frac{\partial \Delta v_{y}}{\partial y}$ of the transverse kick are necessary. We start with Eq. (1) and the complex error function $w(z)$ definition, Eq. (2), to compute its derivative:

$$
\frac{d w}{d z}(z)=\frac{2 i}{\sqrt{\pi}}-2 z w(z) .
$$

$$
\begin{aligned}
& \frac{\partial \Delta v_{x}}{\partial x}(x, y)=\frac{-N K \sqrt{\pi}}{\sigma_{x}^{2}-\sigma_{y}^{2}}\left[\frac{1}{\sqrt{\pi}}-\operatorname{Im}\left[\frac{x+i y}{\sqrt{2\left(\sigma_{x}^{2}-\sigma_{y}^{2}\right)}} w\left(\frac{x+i y}{\sqrt{2\left(\sigma_{x}^{2}-\sigma_{y}^{2}\right)}}\right)\right]+e^{-\left[\frac{x^{2}}{2 \sigma_{x}^{2}}+\frac{y^{2}}{2 \sigma_{y}^{2}}\right]}\left(-\frac{1}{\pi} \frac{\sigma_{y}}{\sigma_{x}}\right.\right. \\
& \left.\left.+\frac{\sigma_{y}}{\sigma_{x}} \operatorname{Im}\left[\frac{x \frac{\sigma_{y}}{\sigma_{x}}+i y \frac{\sigma_{x}}{\sigma_{y}}}{\sqrt{2\left(\sigma_{x}^{2}-\sigma_{y}^{2}\right)}} w\left(\frac{x \frac{\sigma_{y}}{\sigma_{x}}+i y \frac{\sigma_{x}}{\sigma_{y}}}{\sqrt{2\left(\sigma_{x}^{2}-\sigma_{y}^{2}\right)}}\right)\right]+\frac{\sqrt{2\left(\sigma_{x}^{2}-\sigma_{y}^{2}\right)}}{2} \frac{x}{\sigma_{x}^{2}} \operatorname{Im}\left[w\left(\frac{x \frac{\sigma_{y}}{\sigma_{x}}+i y \frac{\sigma_{x}}{\sigma_{y}}}{\sqrt{2\left(\sigma_{x}^{2}-\sigma_{y}^{2}\right)}}\right)\right]\right)\right] \text {. } \\
& \frac{\partial \Delta v_{y}}{\partial y}(x, y)=\frac{-N K \sqrt{\pi}}{\sigma_{x}^{2}-\sigma_{y}^{2}}\left[-\frac{1}{\sqrt{\pi}}-\operatorname{Re}\left[i \frac{x+i y}{\sqrt{2\left(\sigma_{x}^{2}-\sigma_{y}^{2}\right)}} w\left(\frac{x+i y}{\sqrt{2\left(\sigma_{x}^{2}-\sigma_{y}^{2}\right)}}\right)\right]+e^{-\left[\frac{x^{2}}{2 \sigma_{x}}+\frac{y^{2}}{2 \sigma_{y}^{2}}\right]}\left(\frac{1}{\pi} \frac{\sigma_{x}}{\sigma_{y}}\right.\right. \\
& \left.\left.+\frac{\sigma_{x}}{\sigma_{y}} \operatorname{Re}\left[i \frac{x \frac{\sigma_{y}}{\sigma_{x}}+i y \frac{\sigma_{x}}{\sigma_{y}}}{\sqrt{2\left(\sigma_{x}^{2}-\sigma_{y}^{2}\right)}} w\left(\frac{x \frac{\sigma_{y}}{\sigma_{x}}+i y \frac{\sigma_{x}}{\sigma_{y}}}{\sqrt{2\left(\sigma_{x}^{2}-\sigma_{y}^{2}\right)}}\right)\right]+\frac{\sqrt{2\left(\sigma_{x}^{2}-\sigma_{y}^{2}\right)}}{2} \frac{y}{\sigma_{y}^{2}} \operatorname{Re}\left[w\left(\frac{x \frac{\sigma_{y}}{\sigma_{x}}+i y \frac{\sigma_{x}}{\sigma_{y}}}{\sqrt{2\left(\sigma_{x}^{2}-\sigma_{y}^{2}\right)}}\right)\right]\right)\right] .
\end{aligned}
$$

[1] Y. Baconnier, P. F. Tavares, and A. Poncet, Neutralisation of accelerator beams by ionisation of the residual gas, CERN Technical Report No. CERN-PS-94-40-LP, 1994.

[2] G. H. Hoffstaetter and C. Spethmann, Equilibrium ion distribution in the presence of clearing electrodes and its influence on electron dynamics, Phys. Rev. ST Accel. Beams 11, 014001 (2008).

[3] S. Sakanaka, M. Izawa, H. Kobayakawa, and M. Kobayashi, Differences in the ion trapping between uniform and partial bunch fillings, Nucl. Instrum. Methods Phys. Res., Sect. A 256, 184 (1987).

[4] T. O. Raubenheimer and F. Zimmermann, Fast beam-ion instability. I. Linear theory and simulations, Phys. Rev. E 52, 5487 (1995).
[5] L. Wang, J. Safranek, Y. Cai, J. Corbett, R. O. Hettel, T. O. Raubenheimer, J. Schmerge, and J. Sebek, Beam ion instability: Measurement, analysis, and simulation, Phys. Rev. ST Accel. Beams 16, 104402 (2013).

[6] L. Wang, Y. Cai, T. O. Raubenheimer, and H. Fukuma, Suppression of beam-ion instability in electron rings with multibunch train beam fillings, Phys. Rev. ST Accel. Beams 14, 084401 (2011).

[7] W. Cheng, Y. Li, and B. Podobedov, Experimental evidence of ion-induced instabilities in the NSLS-II storage ring, Nucl. Instrum. Methods Phys. Res., Sect. A 861, 38 (2017).

[8] A. Poncet, in Frontiers of Particle Beams: Factories with $e^{+} e^{-}$Rings (Springer, New York, 1994), p. 202. 
[9] G. H. Hoffstaetter and M. Liepe, Ion clearing in an ERL, Nucl. Instrum. Methods Phys. Res., Sect. A 557, 205 (2006).

[10] G. Pöplau, U. van Rienen, and A. Meseck, Numerical studies of the behavior of ionized residual gas in an energy recovering linac, Phys. Rev. ST Accel. Beams 18, 044401 (2015).

[11] H. Takada, Y. Tsutsui, and T. Tomimasu, Ion effects in a compact electron storage ring, Nucl. Instrum. Methods Phys. Res., Sect. A 294, 373 (1990).

[12] Y. Miyahara, K. Takayama, and G. Horikoshi, Dynamical analysis on the longitudinal motion of trapped ions in an electron storage ring, Nucl. Instrum. Methods Phys. Res., Sect. A 270, 217 (1988).

[13] D. Sagan, Some aspects of the longitudinal motion of ions in electron storage rings, Nucl. Instrum. Methods Phys. Res., Sect. A 307, 171 (1991).

[14] A. Gamelin, C. Bruni, and D. Radevych, Nuage, ion cloud tracker, in 8th International Particle Accelerator Conference (IPAC'17), Copenhagen, Denmark, 14-19 May, 2017 (JACOW, Geneva, Switzerland, 2017), p. 3692 .
[15] A. Variola, J. Haissinski, A. Loulergue, F. Zomer et al., ThomX Technical Design Report, Laboratoire de l'Accélérateur Linéaire (LAL), 2014.

[16] R. Talman, in AIP Conference Proceedings (1987), Vol. 153, p. 827.

[17] M. Bassetti and G. A. Erskine, Closed expression for the electrical field of a two-dimensional Gaussian charge, CERN Technical Report, 1980.

[18] A. Gamelin and C. Bruni, Residual ion dynamics in ThomX electron storage ring, in 7th International Particle Accelerator Conference (IPAC'16), Busan, Korea, 2016 (JACOW, Geneva, Switzerland, 2016), p. 1648.

[19] G. Pöplau, A. Meseck, A. Markovik, and U. van Rienen, Simulations for ion clearing in an ERL, in Proceedings of ICAP (2012) pp. 143-145.

[20] N. Hubert, L. Cassinari, J. C. Denard, N. Leclercq, A. Nadji, L. Nadolski, and D. Pédeau, The SOLEIL BPM and orbit feedback systems, in Proc. of the DIPAC (2007).

[21] P. F. Tavares, Bremsstrahlung detection of ions trapped in the EPA electron beam, Part. Accel. 43, 107 (1993).

[22] R. Nagaoka, Ions, CERN Yellow Reports: School Proceedings 3, 519 (2017). 